\title{
Aspetti Fondamentali del Processo Civile di Civil Law e di Common Law
}

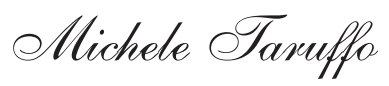

Ordinario di Diritto Processuale Civile nell'Università di Pavia (Italia).

\section{SOMMARIO}

Introduzione;

1 La crisi dei modelli tradizionali;

2 L'evoluzione dei modelli: gli ordinamenti di common law;

3 Segue: gli ordinamenti di civil law;

4 Tendenze di superamento della distinzione;

5 Verso nuovi modelli.

\section{Introduzione}

TU

'analisi comparatistica dei sistemi processuali di civil law e di common law solleva anzitutto alcuni problemi di metodo che conviene affrontare in via preliminare, al fine di collocare nella prospettiva corretta le considerazioni che andrò svolgendo.

Una prima osservazione riguarda in linea generale il metodo della comparazione, ma attiene anche in modo specifico alla comparazione dei sistemi processuali. Senza entrare qui nella discussione sui metodi e le finalità della comparazione giuridica, che si è svolta in Italia ${ }^{1}$ ed anche a livello internazionale, si può sinteticamente dire che pare ormai tramontata l'idea secondo la quale mettere a confronto ordinamenti diversi consisterebbe nella neutrale (e quasi "passiva") misurazione di analogie e differenze tra sistemi normativi, volta allo scopo di stabilire vicinanze e distanze tra

1 Cfr. in particolare i saggi raccolti nel volume L'apporto della comparazione alla scienza giuridica, Milano, 1980. 
norme o tra istituti. Al contrario, si riconosce ormai uniformemente che l'analisi comparatistica si può fare utilmente soltanto sulla base di un "progetto culturale" presupposto e messo in opera da chi effettua l'analisi. ${ }^{2}$ Questa, quindi, non è mai passiva, neutrale o indifferente: è invece condizionata ed orientata sia dalla cultura propria del comparatista, sia dalle finalità in vista delle quali egli svolge il proprio lavoro. Il progetto culturale dello studioso di diritto comparato orienta non solo le sue scelte (su quali ordinamenti, quali istituti, quali esperienze mettere a confronto), ma anche il metodo con cui la comparazione viene svolta. Così, per fare solo qualche esempio, una comparazione basata solo sul raffronto tra norme esistenti in vari paesi in un certo momento è giustificabile soltanto all'interno di una cultura rigorosamente (e restrittivamente) normativistico-positivista, e sulla base dell'idea che la cultura giuridica sarebbe mera giustapposizione di informazioni particolari che si suppone "descrivano" dati normativi (nella quale si dà per scontata una premessa assai problematica, ossia che le norme si possano descrivere). Invece, una comparazione orientata alle riforme ${ }^{3}$ implica la conside- razione dell'effettivo funzionamento dei sistemi e degli istituti, delle sottostanti scelte di politica del diritto, degli orientamenti della prassi e dei principi generali che fondano la validità e l'effettività degli ordinamenti posti a confronto. ${ }^{4}$ In sostanza, rimane vero il tradizionale slogan dei comparatisti per cui il modo migliore di conoscere il proprio ordinamento è quello di conoscere anche altri ordinamenti, ma bisogna considerare che molti e diversi sono i modi per conoscere gli altri ordinamenti (oltre che - naturalmente - per conoscere il proprio).

Un secondo ordine di osservazioni preliminari riguarda ciò che si assume come "oggetto" della comparazione. Il giuristanormativista tradizionale tende a ritenere, come si è già accennato, che si tratti di mettere a confronto "norme". Al più egli è disposto a riconoscere che si debba aver notizia anche di ciò che è indispensabile per intendere almeno superficialmente il significato delle norme, ossia la giurisprudenza e la dottrina. Poichè però conoscere adeguatamente la giurisprudenza e la dottrina di un altro ordinamento (peggio ancora se si tratta di vari altri ordinamenti, magari

2 Di modelli culturali come fondamento e scopo dello studio del diritto comparato parlava già DENTI, Diritto comparato e scienza del processo, in L'apporto della comparazione, cit., p. 212 ss.. In proposito v. da ultimo MONATERI, Critica dell'ideologia e analisi antagonista: il pensiero di Marx e le strategie della comparazione, in Riv.crit.dir.priv. 2000, p. 710 ss., il quale sottolinea opportunamente il venir meno della neutralità pretesa da una parte della dottrina comparatistica.

3 Sul rapporto funzionale tra comparazione e riforme processuali cfr. in particolare DENTI, op. cit., p. 204 ss.; CAPPELLETTI, Dimensioni della giustizia nella società contemporanea, Bologna 1994, p. 25 ss. In proposito si può osservare che, se da un lato lo studio comparatistico può essere utilmente orientato a preparare riforme, dall'altro lato è difficile pensare che buone riforme possano esser fatte senza un'adeguata conoscenza di come altri legislatori hanno affrontato e risolto -magari in modo efficace i medesimi problemi. Questa osservazione sarebbe assolutamente banale se non accadesse in Italia - ma cose analoghe succedono probabilmente anche altrove - che riforme importanti e necessarie vengano confezionate e poste in atto nella più rigorosa $\mathrm{e}$ pervicace ignoranza di ciò che accade in altri ordinamenti. II fatto che riforme provinciali e autarchiche finiscano poi col fallire i loro obbiettivi non può meravigliare, ma questo è un discorso prevalentemente italiano che esula dai confini della presente esposizione.

Cfr. CAPPELLETTI, op. cit., p. 11 ss. 
culturalmente lontani dal proprio) è assai difficile, o addirittura impossibile, ecco che il giurista normativista non si spingerà molto più in là della lettura (magari delegata ad un giovane collaboratore che conosce la lingua) di qualche sintetico e riassuntivo commentario. Se poi si tratta di un processualista tipico ${ }^{5}$, orientato a ritenere che il diritto processuale civile si esaurisca in un insieme di norme tecniche particolari, molto specifiche ed analitiche, ecco che costui tenderà a pensare che la comparazione riguardi micro-problemi, ossia si svolga confrontando norme tecniche di dettaglio, e che l'esito di un lavoro siffatto altro non possa essere che la registrazione delle "variazioni sul tema", ossia delle differenti regole specifiche che i vari ordinamenti adoperano per regolare gli stessi aspetti di dettaglio della procedura. In questo modo la comparazione processuale rimane a livello "micro" e diventa non solo terribilmente noiosa, ma anche sostanzialmente inutile.

Le cose possono configurarsi diversamente, e la comparazione può diventare più interessante e più fruttuosa, se si prendono in considerazione non solo norme specifiche (o norme specifiche prese "da sole"), ma modelli processuali. Non è il caso di approfondire qui la discussione metodologica intorno alla nozione di "modello" : credo basti osservare da un lato che la costruzio- ne di "idealtipi” dei diversi ordinamenti può stabilire un livello di analisi sul quale il confronto è possibile, e produttivo, evitando di perdersi nella massa infinita dei dettagli; dall'altro lato, che si possono costruire modelli di diversa ampiezza e dimensione e con diverso contenuto. Si può pensare, ad es., a modelli del processo di cognizione o del processo cautelare, a modelli probatori, a modelli di organizzazione giudiziaria, come anche a modelli di contenuto assai più ampio o assai più ristretto.

In proposito vale la pena di osservare che la costruzione dei modelli (uso apposta questo termine per sottolineare che il modello non esiste in natura, ma viene appunto - costruito) non può essere arbitraria, se vuole tendere a rappresentare oggetti effettivamente esistenti, ma è comunque opera di colui che studia ed analizza uno o più ordinamenti, e dalla conoscenza di essi trae i "tipi ideali" che appaiono idonei a rappresentare i tratti essenziali di questi ordinamenti. Naturalmente quanto più un modello è approssimato alla realtà che vuole rappresentare, tanto maggiore sarà la sua capacità euristica e la sua utilità come strumento di analisi. Si potrà così parlare di modelli "buoni" o "cattivi", dotati di maggiore $\mathrm{o}$ minore capacità rappresentativa $\mathrm{a}$ seconda della loro "vicinanza" a ciò che viene tipizzato. In ogni caso, tuttavia, la co-

5 Sulle principali caratteristiche di questa figura sia consentito il rinvio a TARUFFO, L'insegnamento accademico del diritto processuale civile, in Riv.trim.dir.proc.civ. 1009, p. 553 ss.

6 Cfr. in proposito i rilievi di CHIARLONI, Nuovi modelli processuali, in Id., Formalismi e garanzie. Studi sul processo civile, Torino 1995, p. 3 ss. Stranamente, nessuna discussione di metodo sull'idea di modello appare invece nell'opera più recente in argomento, ossia GRANDE, Imitazione e diritto: ipotesi sulla circolazione dei modelli, Torino 2000, dato che l'A. si limita ad usare alcuni modelli senza spiegarne il fondamento. 
struzione dei modelli è opera dell'interprete, il quale li costruisce appunto allo scopo di rendere possibile e razionale l'analisi di ordinamenti diversi (o di istituti appartenenti ad ordinamenti diversi). E' proprio qui che emerge l'importanza fondamentale del "progetto culturale" di colui che si pone a svolgere quest'opera, poichè è in funzione di questo progetto che si mettono a confronto alcuni ordinamenti e non altri, si studiano alcuni istituti e non altri, e si scelgono $i$ tratti di quegli ordinamenti ed istituti che si ritengono più importanti e quindi meritevoli di essere inseriti nel "modello ideale" al quale si intende far riferimento. In sostanza, può far parte di qualche progetto culturale non entusiasmante, ma forse utile, l'analisi dei metodi di notificazione degli atti nei sistemi del Sud Est asiatico o lo studio comparato dei termini processuali nei paesi ex-comunisti, mentre rinvia ad un ben diverso progetto culturale, forse non meno utile ma culturalmente più interessante, l'analisi degli strumenti processuali di tutela del consumatore o dei metodi per l'accertamento dei fatti in giudizio nei sistemi occidentali evoluti.

\section{La crisi dei modelli tradizionali}

Proseguendo il discorso sul piano della comparazione di modelli, e venendo al rapporto tra ordinamenti processuali di civil law e di common law, sembra opportuno sgombrare il campo da alcune contrapposizioni tradizionali, che sono state spesso usate per esprimere differenze ritenute fondamentali tra i due tipi di ordinamenti, ma che appaiono da tempo superate e non più capaci di fornire conoscenze attendibili. In realtà, non si tratta di descrizioni completamente false in senso descrittivo, ma di immagini che forse potevano avere qualche significato in passato, e forse sono dotate di qualche efficacia allusiva ancor oggi, ma che sono state indebitamente considerate come essenziali allo scopo di tracciare la distinzione tra $\mathrm{i}$ due tipi di ordinamento processuale.

Indipendentemente dalla loro veridicità rappresentativa, si è trattato di modelli che sono stati costruiti sulla base di precisi progetti culturali, ossia allo scopo di mettere in particolare evidenza - per esaltarne il valore o criticarne la presenza - alcuni aspetti dei vari sistemi processuali che venivano posti a confronto. Oggi la critica che si può rivolgere all'impiego di questi modelli non è tanto che essi forniscano false rappresentazioni della realtà (benchè questo accada piuttosto spesso, se non altro per ignoranza o mancato aggiornamento delle informazioni), quanto che essi erano forse coerenti a scelte di valore o di sistema dirette a privilegiare problemi e soluzioni che ora appaiono di ben minore interesse.

Due esempi consentiranno di rendere il discorso meno astratto, e di verificare l'attendibilità dell'approccio critico che qui si propone. Il primo esempio riguarda la contrapposizione tra oralità e scrittura, secondo la quale il processo di common law sarebbe un processo essenzialmente orale, mentre il processo di civil law sarebbe un processo essenzialmente scritto. Senza entrare qui nel tema generale della 
contrapposizione di principio tra oralità e scrittura, ${ }^{7}$ osservo che la distinzione tra processo orale e processo scritto non ha mai rappresentato, e non rappresenta oggi, la distinzione tra processo di common law e processo di civil law. Per un verso, infatti, è facile osservare che: a) il processo di common law include numerosi atti scritti (probabilmente non meno numerosi di quelli con cui si svolge un processo di civil law), ${ }^{8}$ e questa tendenza è andata rafforzandosi nell'evoluzione più recente; b) il processo di equity, che per secoli ha rappresentato un settore importantissimo del processo inglese, e poi anche di quello nordamericano fino alla fusione con il processo at law, nella quale però si sono conservati numerosi aspetti del processo in equity, era un processo scritto. ${ }^{9}$ L'impiego di prove scritte, poi, non è meno frequente in common law che in civil law, malgrado profonde differenze che riguardano altri aspetti del diritto delle prove.

Per altro verso, si può osservare che in vari ordinamenti di civil law vi sono importantissimi elementi di oralità, che si ritrovano ad es., nelle varie forme di discussione orale, preliminare o conclusiva, della causa, nell'assunzione orale delle prove in udienza, e nella pronuncia orale della decisione che viene prevista in vari casi. Naturalmente qui non si vuole sostenere che procedimenti di common law e procedimenti di civil law non presentino alcuna differenza dal punto di vista dell'alternativa oralità-scrittura; al contrario, nell'estrema varietà della disciplina di molti aspetti del processo queste differenze esistono ed in qualche caso sono molto rilevanti. Ciò che si vuol dire è che risulta sostanzialmente inattendibile un'equivalenza "common law=oralità", così come è inattendibile l'equivalenza "civil law=scrittura", di fronte all'ampia presenza della scrittura nei processi di common law, e all'ampio spazio che all'oralità viene riservato in vari processi di civil law.

Il secondo esempio che qui vale la pena di richiamare è la contrapposizione tra processo adversarial, che si ritiene essere tipico degli ordinamenti di common law, e processo inquisitorial, che si ritiene essere tipico dei sistemi di civil law. ${ }^{10}$ Su questi temi si è nel tempo accumulata, nella cultura giuridica di vari paesi, una letteratura molto ampia, della quale non è qui possibile dar conto in modo esauriente. ${ }^{11} \mathrm{Mi}$ permetterei però una osservazione irrispettosa: molte di quel-

7 Per un panorama comparatistico in argomento cfr. CAPPELLETTI, Procédure orale et procédure écrite, Milano-New York, 1971.

8 II processo di common law conosce numerosi atti scritti con vari nomi (pleadings, writs, briefs, motions, ed altri ancora): sono infatti scritti non solo gli atti introduttivi, ma anche la maggior parte degli atti tramite i quali si svolge la fase di pre-trial: su questi atti cfr. HAZARD-TARUFFO, La giustizia civile negli Stati Uniti, Bologna 1993, p. 125 ss.; TARUFFO, Diritto processuale civile dei paesi anglosassoni, in Dig.disc.priv..Sez.civ., VI, Torino 1990, p. 347 ss.

9 Cfr. SUBRIN, How Equity Conquered Common Law: The Federal Rules of Civil Procedure in Historical Perspective, in 135 U.Pa.L.Rev. 1987, p. 926 ss.; CAPPELLETTI, op. ult.cit., p. 68 ss.

10 Cfr. ad es., con riferimento prevalente al processo penale, GRANDE, op. cit., p. $47 \mathrm{ss}$.

11 Per analisi comparatistiche della contrapposizione in oggetto cfr. per tutti DAMAŠKA, I volti della giustizia e del potere. Analisi comparatistica del processo, tr.it., Bologna 1991, p. 30 ss. e Id., Evidence Law Adrift, New Haven-London 1997, p. 74 ss. In argomento v. anche TARUFFO, II processo civile "adversary" nell'esperienza americana, Padova 1979; Id., Diritto processuale civile, cit., p. 339 ss. 
le pagine sono pura propaganda ideologica a favore dell'uno o dell'altro sistema, e sono completamente inattendibili dal punto di vista scientifico. Mai come su questi temi, infatti, sono emersi all'evidenza - anche se non sempre in modo consapevole - i progetti culturali (o, meno nobilmente, i pregiudizi ideologici) dei giuristi di varie culture che se ne sono occupati. Una considerazione più fredda, e meno ideologicamente condizionata, di questi temi potrebbe infatti portare ad esiti ben diversi, partendo dalla constatazione - formulata da uno dei maggiori comparatisti che oggi operano nell'area del diritto processuale - che la contrapposizione adversarial-inquisitorial, se mai ha avuto davvero un senso (del che è lecito dubitare), è oggi sicuramente logora, superata e sostanzialmente inutile come strumento di analisi. ${ }^{12}$

Per un verso, infatti, si può osservare che i processi di common law sono spesso stati inquisitorial, o comunque nonadversary: anche senza rifarsi al caso classico della Star Chamber inglese, basti ricordare ancora una volta il processo in equity. ${ }^{13}$ Oggi, d'altronde, sono numerosi i tipi di procedimento che non si svolgono affatto secondo il tradizionale modello adversarial, e sono invece caratterizzati da una presenza attiva del giudice. Inoltre, come si vedrà anche più oltre, il processo angloamericano ordinario è oggi caratterizzato dalla presenza di spiccati poteri del giudice, principalmente sul piano della gestione del procedimento.
Per altro verso, si può osservare che (a parte il caso topico della Santa Inquisizione, e senza occuparci qui del processo penale) i processi civili di civil law tutto sono stati o sono, meno che inquisitorial nel senso stretto del termine. Basti considerare che ormai, anche in attuazione di garanzie costituzionali e principi fondamentali, il contraddittorio tra le parti è generalmente assicurato in misura più che soddisfacente. Se "inquisitorio" significa, secondo l'accezione storicamente fondata, che le parti non si possono difendere, allora non esistono in civil law processi che possano propriamente definirsi come inquisitorial. Se invece con questo termine si vuole alludere a modelli di processo in cui il giudice svolge un ruolo attivo nella direzione e gestione del procedimento e il giudice ha autonomi poteri di iniziativa istruttoria, allora la contrapposizione in oggetto cambia di senso, ma rimane ugualmente in larga misura infondata. Basta pensare all'amplissima, lunga ed importante esperienza del processo liberale classico, ${ }^{14}$ imperniato su un'attuazione intensa ed onnipervasiva del principio dispositivo, per rendersi conto che nulla è stato più estraneo, alla storia del processo civile di civil law, di un modello davvero inquisitorio di processo civile. Quanto ai poteri istruttori del giudice, è noto che anche oggi i vari sistemi di civil law adottano orientamenti molto diversi, anche in funzione della diversità dei procedimenti

12 Cfr. DAMAŠKA, I volti della giustizia, cit., p. 34.

13 V. riferimenti supra, nella n.9, cui adde TARUFFO, Diritto processuale civile, cit., p. 327; CAVANNA, Storia del diritto moderno in Europa.I. Le fonti e il pensiero giuridico, Milano, rist.1982, p. 532 ss.

14 Per più ampie osservazioni in proposito, con riferimento al cod.proc.civ. italiano del 1865, sia consentito il rinvio a TARUFFO, La giustizia civile in Italia dal 700 ad oggi, Bologna 1980, p. 142 ss. 
riferiti a specifiche materie (come nel processo del lavoro in Italia), sicchè l'affermazione per cui il modello di civil law sarebbe caratterizzato da forti poteri istruttori ufficiosi risulta inattendibile, e per di più nasconde dietro una generalizzazione indebita problemi assai complessi, che trovano soluzioni molto diverse nei vari ordinamenti.

\section{L'evoluzione dei modelli: gli ordinamenti di common law}

Il fatto è che, rispetto alle situazioni nelle quali sono stati elaborati gli schemi fondati sulla contrapposizione oralità-scrittura e adversarial-inquisitorial, molti mutamenti sono avvenuti nei diversi ordinamenti. Ciò non implica alcuna convalidazione storica ex post di queste contrapposizioni, in quanto non dimostra che esse fossero vere e ideologicamente neutre quando sono state elaborate, ma implica comunque che queste categorie siano ormai invecchiate e non servano a segnalare gli aspetti più rilevanti dei sistemi processuali attuali. Ciò ha determinato anche una trasformazione dei paradigmi che appaiono più utili per interpretare questi sistemi: il progetto culturale consistente nel cercare di stabilire - onde prospettarne opportunità e vantaggi - quali sistemi fossero improntati all'oralità, essenzialmente al fine di deprecare quelli che apparivano ancora legati al metodo della scrittura, ${ }^{15}$ sembra aver fatto il suo tempo. Innovazioni nel senso della scrittura (come nel caso delle attestations francesi, ${ }^{16}$ o dell'eliminazione dell'udienza di discussione in Italia) ${ }^{17}$ vengono introdotte senza eccessivo scandalo: evidentemente la sensibilità verso questo genere di problemi si è molto attenuata. Anche il progetto culturale imperniato sull'esaltazione del modello adversarial e sulla denuncia degli aspetti inquisitori del processo civile sembra aver fatto il suo tempo, nella dottrina nordamericana come in quella dei paesi di civil law. Forse ci si è resi conto del fatto che non è più tempo di rigide contrapposizioni dogmatiche e di contrasti di principio, o che non è più tempo di insistere su quelle contrapposizioni e su quei contrasti, poichè sono emersi ben altri problemi nel campo della giustizia civile, e molti legislatori hanno assunto orientamenti di riforma più pragmatici e meno ideologicamente orientati. In ogni caso, se non si vuole chiudere ad ogni costo la realtà fuori della porta della scienza giuridica, occorre tener conto delle molte ed importanti trasformazioni che si sono verificate e che sono tuttora in corso - in molti ordinamenti processuali di common law e

15 Questo progetto era sotteso, ad esempio, all'opera fondamentale di CAPPELLETTI su La testimonianza della parte nel sistema dell'oralità 2 voll., Milano 1962), ma lo si ritrova anche alla base dei riferimenti comparatistici che CHIOVENDA portava a sostegno della sua opera di propaganda a favore del principio di oralità (v. infatti Lo stato attuale del processo civile in Italia e il progetto Orlando di riforme processuali, ora in Id., Saggi di diritto processuale civile (1894-1937), vol.I, Milano 1993, p. 395 ss.).

16 Cfr. gli artt. 200 ss.del code de procédure civile.

17 Cfr. gli attuali artt. 275 e 281 quinquies cod.proc.civ. (mentre pare destinato a rimanere inapplicato l'art.281 sexies, che prevede la possibilità di trattazione orale con contestuale pronuncia orale della sentenza motivata in udienza). Non a caso nel giustificare la eliminazione sostanziale dell'udienza di discussione PROTO PISANI, La nuova disciplina del processo civile, Napoli 1991, p. 172, la definisce una "inutile farsa". 
di civil law. E' tenendo conto degli esiti di queste trasformazioni, infatti, che si potranno costruire nuovi modelli utili per l'analisi comparatistica dei sistemi processuali.

Variazioni importanti sono avvenute negli ultimi anni nei principali sistemi processuali di common law, tali da segnare significative trasformazioni di questi ordinamenti (e la crisi, di cui si è già fatto cenno, dei relativi modelli tradizionali). Naturalmente non è possibile dar conto in questa sede di tutte queste trasformazioni, che richiederebbero estese analisi ad hoc. Qualche sintetico riferimento può però essere sufficiente a mostrare come il modello di common law abbia perduto o stia perdendo alcuni dei suoi connotati tradizionali e ne abbia acquisiti di nuovi. Farò qui riferimento a tre aspetti molto importanti: a) il ruolo del giudice; b) la natura e funzione della fase di pre-trial"; c) il ruolo della giuria.

a) Nel tradizionale modello adversarial del processo la figura del giudice era quella di un "arbitro passivo", di un umpire disinformato e disinteressato, oltre che neutrale, che aveva la sola ed esclusiva funzione di assistere al libero scontro delle parti garantendone la correttezza e sanzionando i comportamenti unfair o illeciti. ${ }^{18}$ Questa immagine mitica è stata travolta in modo pressochè completo ed è stata sostituita da un'immagine del giudice molto diversa. In Inghilterra questa trasformazione viene portata a compimento con le Civil
Procedural Rules 1998 entrate in vigore il 26 aprile 1999. Si tratta di un vero e proprio codice di procedura civile ${ }^{19}$ (un'altra innovazione rilevantissima rispetto all'immagine tradizionale del diritto processuale civile inglese come diritto non scritto, che peraltro già da tempo era diventata inattendibile), nel quale al giudice vengono affidati numerosi ed incisivi poteri di governo e direzione del procedimento, come quello che attiene alla determinazione del track che la causa dovrà seguire (ossia del tipo di procedimento applicabile a seconda della natura della causa), alla fissazione delle udienze e all'acquisizione delle prove. ${ }^{20}$

Negli Stati Uniti una trasformazione analoga si ha, a partire dagli anni '70, con l'emersione della figura del managerial judge, che prende il posto dell'immagine tradizionale del giudice come passive umpire. Si tratta di una trasformazione lunga e complessa, che non ha un preciso punto di riferimento temporale come le Rules inglesi, ed è la conseguenza di vari fattori come la continua riforma delle Federal Rules of Civil Procedure, le riforme decentrate e localizzate alle quali apre la strada il Civil Justice Reform Act del 1990, e le risposte che la prassi giudiziaria dà ai crescenti problemi di gestione e controllo della giustizia civile, in particolare con riferimento alla fase preliminare del processo (su cui infra, sub b). In ogni caso, quando Judith Resnick, in un saggio giustamente

18 Cfr., anche per riferimenti, DAMAŠKA, I volti della giustizia, cit., p. 231 ss.; Id., Evidence Law Adrift, cit., p. 76 ss.; TARUFFO, Diritto processuale civile, cit., p. 341; Id., II processo civile "adversary", cit., p. 117 ss.

19 Cfr. CPR, rule 1.1, ove le stesse Rules sono espressamente definite come "a new procedural code".

20 Nella letteratura italiana in argomento cfr., anche per ampi riferimenti bibliografici, PASSANANTE, La riforma del processo civile inglese: principi generali e fase introduttiva, in Riv.trim.dir.proc.civ. 2000, p. 1363 ss., 1378 ss. 
famoso del $1982^{21}$ fotografa il managerial judge e ne analizza l'origine e le caratteristiche principali, questa trasformazione è già sostanzialmente compiuta: spetta al giudice non più il ruolo dell'arbitro passivo che si limita a "fischiare i falli" commessi dalle parti, ma un ruolo di organizzazione e gestione attiva, e non solo di controllo, dello svolgimento del processo. ${ }^{22}$

Se poi si guarda in particolare ai poteri di iniziativa istruttoria del giudice si constata che, con buona pace dell'immagine tradizionale del giudice angloamericano completamente privo di siffatti poteri, in entrambi gli ordinamenti il giudice può svolgere anche un ruolo marcatamente attivo sul piano delle iniziative probatorie. Rilevanti poteri del giudice in materia di prove sono stati infatti previsti nelle nuove Rules inglesi, ${ }^{23}$ e significativi poteri istruttori erano già stati previsti nelle Federal Rules of Evidence statunitensi introdotte nel 1975 ed emendate nel 1994 , in particolare con riferimento alla prova testimoniale e alla expert evidence. ${ }^{24}$

b) Limmagine storicamente risalente e diffusa del processo angloamericano è quella di un procedimento incentrato su un'udienza di tipo dibattimentale nella quale si assumono le prove testimoniali, gli avvocati discutono oralmente la causa, e subito dopo viene pronunciata la sentenza. ${ }^{25}$ Tuttavia, per varie ragioni di funzionalità del trial sin dall'inizio del '900 si sono venute configurando, in Inghilterra come negli Stati Uniti, fasi preliminari (pre-trial) con la funzione di consentire alle parti di prepararsi adeguatamente al dibattimento, essenzialmente tramite la discovery delle prove in possesso dell'avversario o di un terzo. ${ }^{26} \mathrm{Di}$ qui l'immagine consolidata del processo di common law come procedimento scisso in due fasi: una fase di pre-trial con funzione essenzialmente preparatoria, ed una fase di trial per l'assunzione delle prove orali in udienza attraverso il meccanismo della direct e cross examination. Questo modello regge ancora come rappresentazione approssimativa del processo angloamericano, ma bisogna osservare che ormai esso rischia di produrre fraintendimenti ed errori se viene considerato come una fedele descrizione di ciò che normalmente accade nelle corti inglesi o americane. Al di là del modello teorico bifase, infatti, la realtà si è evoluta in maniera largamente diversa. Si tratta di

21 Cfr. RESNICK, Managerial Judge, in 96 Harv.L.Rev. 1982, p. 376 ss.. Sul mutamento della funzione del giudice provocato dal diffondersi della public law litigation va ricordato anche il classico saggio di CHAYES, The Role of the Judge in Public Law Litigation, in 89 Harv.L.Rev. 1976, p. 1284 ss.

22 Nella letteratura recente in proposito cfr. ad es. SCHWARTZER, Case Management in the Federal Courts, in C.J.Q. 1996, p. 141 ss., ed in particolare Just, Speedy and Inexpensive? An Evaluation of Judicial case Management Under the Civil Justice Reform Act, Rand Co., Santa Monica, Ca., 1996. Cfr. CPR, rule 32.1. (1)(a)(b)(c) e 32.1.2.

24 Cfr. la Rule 614(a), che prevede il potere d'ufficio del giudice di disporre prove testimoniali non dedotte dalle parti, la Rule 614(b), secondo la quale il giudice può interrogare i testimoni dedotti dalle parti, e la Rule 706 relativa al potere del giudice di nominare consulenti tecnici d'ufficio.

25 Cfr.TARUFFO, Diritto processuale civile, cit., p. 347 ss., 367 ss.; HAZARD-TARUFFO, op. cit., p. 151 ss.; JACOB, La giustizia civile in Inghilterra, tr.it., Bologna 1995, p. 147 ss.

Cfr. TARUFFO, ibidem, p. 348, 353 ss., 360 ss. 
fenomeni non nuovi, che tuttavia vale la pena di ricordare. In Inghilterra accade da decenni che una percentuale bassissima di controversie civili (dell'ordine del 2-3\% delle cause iniziate) sopravviva alla fase preliminare e giunga al trial per poi concludersi con una sentenza. Questo fenomeno si verifica per varie ragioni che non possono essere qui analizzate: va tuttavia segnalato il fatto che il legislatore inglese ha in vari modi incoraggiato questa tendenza, spingendo decisamente nel senso di favorire la risoluzione precoce della lite nella fase di pre-trial. Diverse tecniche, che includono tentativi di conciliazione, decisioni in default, giudizi sommari e payments into court vengono adoperate per conseguire questo scopo. ${ }^{27}$

Negli Stati Uniti la situazione normativa e le tecniche usate sono in parte diverse, ma non è sostanzialmente diverso il risultato: una percentuale elevatissima di cause civili non supera infatti la fase di pretrial e non giunge al dibattimento, nella maggior parte dei casi perchè le parti concordano un settlement o perchè il giudice ha disposto un tentativo di conciliazione presso un terzo o un esperimento arbitrale, o perchè ha avuto esito qualche altro meccanismo di soluzione precoce della controversia. $^{28}$

Questi fenomeni sono importantissimi da molti punti di vista che qui non possiamo analizzare in modo adeguato, ma producono almeno due conseguenze "di sistema" che vanno messe in evidenza. La pri- ma conseguenza è che in processi così congegnati, nei quali le sorti della grandissima maggioranza delle cause si giocano nella fase preliminare, la contrapposizione adversarialinquisitorial diventa completamente priva di senso. Ciò che accade, infatti, è che nell'ambito del pre-trial si sviluppa una serrata dialettica tra difensori, e tra difensori e giudice, che non è adeguatamente analizzabile nei termini di questa contrapposizione. La seconda conseguenza è che il modello del processo di common law non può più essere definito secondo lo schema bifasico di pretrial e trial, se non a costo di provocare gravi malintesi, per l'ottima ragione che in una frequenza dell'ordine del 90-98\% dei casi il trial non ha luogo. Diventa addirittura improprio includere nel modello la pronuncia della sentenza a seguito dell'assunzione delle prove, dato che raramente - ossia solo quando si è svolto il trial - il processo si conclude con la sentenza. In sostanza ed in sintesi: paradossalmente la fase dipre-trial, nata con funzione preparatoria - ossia per consentire alle parti di giungere al trial nelle migliori condizioni per giocare in esso le proprie carte difensive- serve davvero a preparare il dibattimento solo quando funziona male, ossia nei rari casi in cui falliscono tutti i meccanismi predisposti per far sì che la causa si chiuda al più presto. Il pre-trial, dunque, si configura essenzialmente come una fase di "risoluzione della controversia senza decisione”, e solo eccezionalmente svolge davvero una funzione preparatoria. ${ }^{29}$

27 Cfr. in particolare JACOB, op. cit., p. 77 ss.

28 Cfr. HAZARD-TARUFFO, op. cit., p. 130 ss.

29 Cfr. in particolare GROSS-KENT, Don't Try: Civil Jury Verdicts in a System Geared to Settlement, in 44 UCLA L.Rev. 1996, p. 1 ss. 
Se si tiene conto di tutto ciò, è facile concludere che il modello tipico del processo di common law è ora costituito essenzialmente da una fase, nella quale entrambe le parti, sotto l'attiva direzione del giudice, chiariscono i termini della controversia, acquisiscono tramite la discovery informazioni sulle rispettive difese e sulle prove che potrebbero essere impiegate, valutano l'opportunità di un accordo o di una rinuncia a proseguire il processo, e transigono o si servono di uno degli altri mezzi di risoluzione rapida della controversia. E' da osservare che questa fase del procedimento è essenzialmente scritta, è diretta dal giudice che dispone al riguardo di ampi poteri, e - a differenza dell'immagine tradizionale del trial - non è neppure concentrata, poichè anzi si svolge in passaggi che possono anche essere numerosi e complicati, e possono richiedere - almeno nei casi più complessi tempi assai lunghi. Come si è detto, solo nei rari casi in cui questo procedimento, che è diventato il vero processo, non riesce a por fine alla controversia, avrà luogo il dibattimento per l'assunzione delle prove e verrà pronunciata una sentenza.

c) Un terzo fattore che tradizionalmente si è considerato come caratteristico del processo di common law è la presenza della giuria nelle controversie civili. Anche a questo proposito, tuttavia, si sono verificati mutamenti di grande importanza. In
Inghilterra la giuria civile è scomparsa ormai da decenni, sicchè sarebbe errato immaginare il processo civile inglese come incentrato sul dibattimento di fronte alla giuria. Anche nei pochissimi casi in cui, come si è appena visto, il processo arriva al dibattimento, questo si svolge di fronte ad un giudice togato (monocratico), senza alcuna giuria. ${ }^{30}$

Negli Stati Uniti si è verificato un fenomeno sostanzialmente analogo, ${ }^{31}$ benchè in termini meno assoluti: la giuria civile è ancora presente, infatti, in una percentuale di casi non trascurabile, anche se nettamente minoritaria $^{32}$. Le ragioni principali di questa sopravvivenza sono sostanzialmente due: il fatto che il jury trial sia previsto come garanzia dal $7^{\circ}$ Emendamento della Costituzione americana, ${ }^{33}$ ma soprattutto il fatto che le giurie popolari sono famose per la loro generosità nell'accordare risarcimenti e punitive damages che talvolta raggiungono somme elevatissime, sicchè l'attore in una causa di responsabilità per danno può essere indotto ad affrontare i costi elevati, ed anche i rischi, del jury trial, nella speranza di vincere molto denaro alla lotteria giudiziaria. Se tuttavia non si sopravalutano fenomeni di questo genere, che appartengono quasi più al folklore giudiziario (benchè talvolta miliardario) che alla prassi prevalente, si può concludere che anche nel sistema statunitense sarebbe improprio configurare la giuria come un elemen-

30 Cfr. JACOB, op. cit., p. 154 ss.

31 Sul declino del trial by jury in Inghilterra e negli Stati Uniti cfr. in particolare DAMAŠKA, Evidence Law Adrift, cit., p. 126 ss.

32 Nella percentuale molto ridotta di casi che vanno al trial per essere decisi con sentenza, la giuria risulta essere presente con una frequenza di circa l' $80 \%$ delle cause di risarcimento dei danni: cfr. KIRKPATRICK, Evidence Law in the Next Millennium, in 49 Hastings L.J. 1998, p. 365 ss.

33 La concezione garantistica della giuria spiega l'estensione del jury trial, avvenuta nel 1991, ai casi di discriminazione in materia di lavoro (42 U.S.C. $\$ 1981 \mathrm{a}(\mathrm{c})(1991))$. 
to fondamentale del modello di processo civile.

\section{Segue: gli ordinamenti di civil law}

Se ora si guarda al mondo degli ordinamenti di civil law, il problema dei modelli processuali si presenta ancor più complesso, ad un punto tale che sembra davvero impossibile discutere sensatamente di un modello di civil law. Riducendo a poche battute un discorso che richiederebbe un'approfondita analisi comparatistica, si possono sottolineare due aspetti particolarmente importanti: a) in realtà non è mai esistito un modello omogeneo ed unitario di processo civile di civil law; b) negli ultimi decenni si sono verificate tante e tali trasformazioni in vari ordinamenti processuali dell'area civilistica, che si è probabilmente perduta ogni possibilità di far riferimento in modo sintetico e unitario ai modelli tradizionali.

a) Storicamente, se si pensa ai secoli del diritto comune, nell'Europa con- tinentale non è mai esistito un modello omogeneo di processo civile. La disciplina del processo era anzi estremamente frammentata in funzione della diversità delle giurisdizioni e della pluralità delle fonti regolatrici della procedura, sicchè non vi era molto in comune, ad es., tra i processi disciplinati dal Code Louis, ${ }^{34}$ dagli statuti italiani, ${ }^{35}$ dalla prassi giudiziaria della Rota romana e degli altri Grandi Tribunali, ${ }^{36} \mathrm{O}$ dalle Kammergerichtsordnungen germaniche. ${ }^{37} \mathrm{Il}$ consueto riferimento al processo romano-canonico come base costante degli ordinamenti processuali continentali non è fondamentalmente errato, se serve a segnalare alcuni caratteri molto generali del processo civile di diritto comune come la scrittura, la durata e la non concentrazione, ma non può nascondere le grandi e profonde differenze che hanno segnato per secoli questi ordinamenti. Ancora nel '700 vi è ben poco in comune tra il Codex Fridericianus Marchicus in Prussia, ${ }^{38}$ il codice "giuseppino" in Austria, ${ }^{39}$ le Costituzioni piemontesi ${ }^{40}$ e gli

34 Cfr. Code Louis. T.I. Ordonnance civile (1667), rist. Milano 1996, su cui cfr. PICARDI, II giudice e la legge nel Code Louis, in Riv.dir.proc. 1995, p. 33 ss.

35 Cfr. SELLA, II procedimento civile nella legislazione statutaria italiana, Milano 1927, e cenni in TARUFFO, La giustizia civile, cit, p. 8 ss.

36 Cfr. GORLA, I Tribunali Supremi degli Stati Italiani, fra i secc.XVI e XIX, quali fattori della unificazione del diritto nello Stato e della sua uniformazione fra Stati (Disegno storico-comparativo), in La formazione storica del diritto moderno in Europa, Firenze 1977, I, p. 447 ss.; Id., I "Grandi Tribunali" italiani fra i secoli XVI e XIX: un capitolo incompiuto nella storia politico-giuridica d'Italia, in Quad.Foro It. 1969, c.629 ss.

37 II riferimento è agli atti fondamentali di istituzione (1495) e di riforma (1555) del Reichskammergericht. Sul processo presso questo organo è fondamentale la ricerca di RANIERI, Recht und Gesellschaft im Zeitalter der Rezeption. Eine rechts- und sozialgerichtliche Analyse der Tätigkeit des Reichskammergerichts im 16. Jahrhundert,I, Köl-Wien 1985, specialmente p. 195 ss.. Cfr. inoltre l'ampia bibliografia citata ivi, I,p. 19 ss.

38 Cfr. Codex Fridericianus Marchicus. T.I, Project des Codicis Fridericiani Marchici, 1748, rist. Milano 2000, e ivi, p. XI ss., la presentazione di H.Mohnhaupt.

39 Cfr. Regolamento Giudiziario di Giuseppe II.1781, rist. Milano 1999, ed ivi la Prefazione di N.Picardi (pubblicata anche in Riv.dir.proc. 2000, p. 36 ss.) e l'Introduzione di W.Ogris e P. Oberhammer.

40 Cfr. VIORA, Le costituzioni piemontesi (Leggi e costituzioni di S.M. il Re di Sardegna). 1723-1729-1770, Torino 1928, e cenni in TARUFFO, op. ult.cit., p. 19 ss. 
statuti e le prassi giudiziarie in vigore in una infinità di stati e staterelli in tutta Europa. Nel corso del secolo XIX sembra verificarsi una convergenza dovuta all'imitazione, che si diffonde in Europa, del modello costituito dal code de procédure civile napoleonico, ${ }^{41}$ ma questa convergenza viene meno verso la fine del secolo essenzialmente a causa della rottura determinata dalla Zivilprozessordnung austriaca, che si pone come nuovo e diverso oggetto di ispirazione e di imitazione per gran parte della dottrina e per alcuni legislatori processuali successivi. ${ }^{42}$ Se poi si guarda al '900 si scopre che l'area del processo di civil law conosce almeno tre modelli principali: quello francese, che continua ad essere seguito in alcuni paesi come ad es. il Belgio e l'Italia; quello austro-tedesco, che viene seguito anche nei paesi scandinavi e in quelli dell'Europa Orientale, oltre che in Giappone, e quello spagnolo, che viene seguito in molti paesi dell'America Latina. Non è il caso di approfondire qui l'analisi di questi modelli, delle loro variazioni e degli adattamenti che si sono verificati a più riprese nei diversi ordinamenti: anche una considerazione superficiale come quella che si è qui proposta basta a mostrare come sia destituita di fondamento, anche sotto il profilo storico, l'idea che in passato sia esistito, e tanto meno che esista oggi, un modello unitario ed omogeneo di processo nell'area dei sistemi di civil law. b) Se si considera il panorama che si è venuto delineando negli ultimi anni all'interno di quest'area si possono riscontrare varie ulteriori ragioni per escludere l'esistenza effettiva di siffatto modello. Non è ovviamente possibile svolgere qui un'analisi comparatistica soddisfacente, ma qualche esempio può bastare ad illustrare questo punto. Da un lato, occorre considerare che all'interno del mondo di civil law si vanno manifestando ormai da tempo forti influenze derivanti dai modelli di common law, ed in particolare dal modello nordamericano, per una serie di ragioni storiche, politiche e culturali. Un esempio molto significativo è rappresentato dal Giappone: è noto che l'ordinamento processuale giapponese si è ispirato al modello germanico a partire dalla fine dell" 800 , sicchè da allora il Giappone è appartenuto a pieno titolo al novero dei sistemi processuali di tipo europeo. ${ }^{43} \mathrm{Pe}$ raltro, dopo la fine della seconda guerra mondiale l'influenza americana si è manifestata in modo molto intenso, sicchè il codice di procedura civile giapponese entrato in vigore nel 1998 delinea un sistema "misto", nel quale elementi di origine nordamericana (come ad es. la cross examination) si combinano con residui elementi di origine germanica (soprattutto con riguardo allo schema del procedimento), e con elementi autoctoni (in particolare la conciliazione).

41 Sull'influenza del codice processuale francese sui codici italiani dell" 800 v. TARUFFO, op. ult.cit., p. 55 ss.

42 A proposito dell'influenza esercitata dal codice austriaco sul pensiero di Chiovenda cfr. TARELLO, L'opera di Giuseppe Chiovenda nel crepuscolo dello Stato liberale, in Materiali per una storia della cultura giuridica 1973, p. 699 ss.

43 Cfr. in particolare i saggi di NAKAMURA raccolti nel volume Japan und das deutsche Zivilprozessrecht - Sammelband der zivilprozessualen Abhandlungen, Tokyo 1996. 
Altre influenze nordamericane si possono riscontrare facilmente in vari ordinamenti di civil law: basti pensare alla recezione di forme di tutela giurisdizionale degli interessi diffusi modellate sull'esempio della class action (recezione che è avvenuta o sta avvenendo, oltre che in Brasile, in altri paesi di civil law). ${ }^{44}$ Questi esempi servono anche a richiamare l'attenzione sul fatto che variazioni ed interferenze non si verificano soltanto al livello dei modelli globali di procedimento: sono anzi più frequenti, e non meno importanti, gli scambi, le interferenze e i "trapianti" che riguardano singoli istituti processuali.

Dall'altro lato vale la pena di considerare che anche all'interno dei sistemi di civil law si sono verificate trasformazioni di grande importanza, che hanno comportato mutamenti globali dei paradigmi processuali precedenti. Due esempi possono bastare ad illustrare questo punto. Il primo esempio riguarda i sistemi processuali di lingua tedesca. La Zivilprozessordnung germanica del 1877 risentiva direttamente dell'influenza del modello francese, ma ciò non avveniva in Austria, dove la disciplina del processo civile aveva seguito un'autonoma linea evolutiva con i codici del 1781 e del $1815 .{ }^{45}$ Comunque, il codice di Klein del 1895 non solo innovò radicalmente la situazione della giustizia civile in Austria, ma costituì il modello per le successive riforme germaniche che dopo varie tappe hanno messo capo principalmente alla Beschleunigungsnovelle del 1977. In queste trasformazioni si verifica per un verso l'adozione del modello di processo orale e concentrato delineato da Klein, che rappresenta un'alternativa radicale rispetto al modello francese (e italiano) del processo scritto e non concentrato. Per altro verso, tuttavia, si manifesta in qualche misura anche l'influenza del modello "classico" di common law, ossia dello schema procedimentale bifasico, composto da una fase preparatoria e da una fase dibattimentale di assunzione delle prove. Non è un caso che, se si esamina ora lo schema strutturale fondamentale del processo germanico (e quello, per molti versi simile, che si ritrova in vari paesi scandinavi), si possono rinvenire più analogie "di sistema" di questo processo con lo schema del processo angloamericano, di quante se ne possano trovare con il processo francese $\mathrm{O}$ italiano. In questo modo il tradizionale "ordine dei modelli" fondato sulla distinzione globale tra civil law e common law appare profondamente modificato: il modello a due fasi esiste ormai in molti sistemi di common law ed anche in vari sistemi di civil law, nonchè in vari sistemi misti come quello giapponese.

Il secondo e più recente esempio è fornito dalla Ley de Enjuiciamiento Civil spagnola che è entrata in vigore all'inizio del 2000 e viene applicata dall'inizio del 2001. La Spagna, come si è già accennato, era rimasta al di fuori o ai margini dell'influenza del modello francese: il codice processuale del 1881, infatti, non rientrava a pieno ti-

44 In proposito cfr. GIUSSANI, Studi sulle "class actions",Padova 1996.

45 Cfr. TARUFFO, op. ult.cit., p. 70 ss. 
tolo nel novero delle variazioni su questo modello. La situazione cambia radicalmente nel 2000, ma non perchè viene tardivamente recepito il modello francese, bensì in quanto il legislatore spagnolo adotta - per quanto riguarda la struttura del procedimento - lo schema bifasico che era andato consolidandosi - come si è appena visto - in Austria e in Germania, ma che ha mostrato di avere una notevole forza di espansione anche al di fuori degli ordinamenti di lingua tedesca. Con ciò il legislatore spagnolo non è uscito dall'area di civil law, ma è certo che l'ordinamento spagnolo attuale non rientra nell'area di influenza del modello francese e quindi finisce col non aver molto in comune, ad es., con l'ordinamento italiano. ${ }^{46}$

Come si vede, dunque, l'idea di un modello omogeneo del processo di civil law è stata e rimane una fictio concettuale che ha nascosto e confuso la realtà effettiva dei principali ordinamenti dell'Europa continentale e di quelli che ne sono in vario modo derivati in Europa e in altre parti del mondo. Si è finito col sovrapporre a questa realtà diversificata un modello teorico unitario che non solo era piuttosto vago ed indeterminato nei suoi lineamenti fondamentali, ma che per di più non trovava reale corrispondenza in alcun tipo di effettiva uniformità delle discipline processuali che pretendeva di descrivere. Non è agevole individuare le ragioni della diffusione e del successo di questo malinteso culturale, che pure è stato - ed in larga misura è tuttora - piuttosto comune. Forse, ma si tratta di un'ipotesi tutta da verificare, si è costruita una omogeneità largamente fittizia per avere qualcosa da contrapporre a ciò che non si conosceva e di cui si temeva l'intrusione, ossia al processo angloamericano. In fondo non va dimenticato che anche oggi, e da parte di molti giuristi non soltanto italiani, anglicum non legitur, e che normalmente si diffida di ciò che non si conosce.

Peraltro, non va dimenticato che nella cultura di common law si è verificato il fenomeno simmetrico: ad un'immagine fortemente idealizzata e ideologizzata del processo adversary come modello ideale del processo angloamericano si è contrapposta un'immagine altrettanto ideologizzata del processo inquisitorial come modello tipico del processo europeo, senza avvertire che nessuna delle due immagini corrispondeva alla realtà effettiva. Che questo fosse dovuto ai limiti della cultura giuridica americana pare evidente: in ogni caso, si è ricondotto il mondo degli ordinamenti processuali di civil law ad uno schema fortemente semplificato e connotato negativamente, forse per giustificare la mancata conoscenza di questo mondo e la scarsa volontà di impegnarsi per conoscerlo.

\section{Tendenze di superamento della distinzione}

Le considerazioni che precedono non sono dirette a sostenere che sia venuta

46 Su questi aspetti del nuovo processo spagnolo cfr., nell'ormai ricca letteratura in argomento, de la OLIVA SANTOS e DIEZPICAZO GIMENEZ, Derecho procesal civil. El proceso de declaraciòn, Madrid 2000, pp. 11 ss., 563 ss. 
meno ogni differenza tra i sistemi processuali di common law e i sistemi di civil law: una conclusione del genere sarebbe evidentemente assurda di fronte alle numerose e rilevanti differenze che tuttora sussistono. Ciò che si vuole suggerire è che non sono più accettabili i termini tradizionali nei quali la distinzione tra i due gruppi di ordinamenti è stata per molto tempo formulata in quanto, ammesso che i due modelli abbiano mai avuto qualche capacità descrittiva, la realtà normativa - ed ancor più quella dell'esperienza effettiva - di questi sistemi appare profondamente mutata già ora, ed appare destinata ad ulteriori profonde trasformazioni. Mentre viene meno l'apparente chiarezza dei due modelli e della loro distinzione, il panorama degli ordinamenti processuali attuali cambia profondamente anche a causa dell'emersione e della crescente importanza di tendenze che non possono più essere ricondotte entro la statica classificazione di ogni singolo sistema processuale come "di common law", "di civil law" o "misto". Assumono inoltre grande rilevanza ordinamenti che in passato venivano lasciati al margine (o al di fuori) del panorama che si prendeva in considerazione ma che ora, e più ancora nel prossimo futuro, non possono più essere trascurati. Valga per tutti l'esempio della Cina, che non solo ha ormai acquisito un ruolo di primo piano sotto il profilo politico ed economico, ma sta anche riformando a varie riprese il sistema di amministrazione della giustizia seguendo percorsi che appaiono difficilmente riconducibili entro le consuete tassonomie occidentali, anche se i tratti fondamentali di quel sistema consentono di ricondurlo approssimativamente all'area di civil law. ${ }^{47}$

Una situazione così complessa, nella quale residui ancora importanti dei modelli tradizionali convivono con novità che si sviluppano rapidamente, non può essere qui analizzata con descrizioni che pretendano di essere complete, e che sarebbero comunque destinate all'obsolescenza in tempi relativamente brevi. Vale però la pena di accennare sommariamente ai fattori dinamici che sembrano essere all'opera nel contesto dei mutamenti relativi ai principali sistemi processuali.

Un primo fattore di notevole rilievo è quello che i comparatisti usano denominare circolazione dei modelli. A differenza di quanto è accaduto per secoli, quando gli ordinamenti processuali mutavano di regola (anche se non mancano eccezioni come la Rezeption germanica) con ritmi molto lenti ma soprattutto "in verticale", ossia ognuno rimanendo entro se stesso ed evolvendo secondo proprie linee storiche, il fenomeno che caratterizza l'evoluzione più recente è quello delle "interferenze orizzontali" tra sistemi diversi, o - se si preferisce - dell'imitazione di un sistema o modello da parte di altri sistemi, anche se derivati da esperienze storiche e linee evolutive molto eterogenee. Ciò che qui si vuole sottolineare è che per quanto riguarda il processo civile questi fenomeni si verificano da qualche tempo non solo tra singoli ordinamenti processuali statali, ma anche tra modelli generali o aree di ordinamenti (potendo

47 Per la traduzione italiana del codice di procedura civile cinese e di varie leggi attinenti all'amministrazione della giustizia cfr. Ricerche sul processo. 7. Il processo civile cinese, Rimini 1998, con saggi introduttivi e di commento di vari autori. 
comunque riguardare, a seconda dei casi, schemi procedimentali complessivi oppure singoli istituti o specifici remedies). Si verifica cioè un complesso interscambio di modelli anche tra sistemi di common law e sistemi di civil law. Gli esempi sono numerosissimi, soprattutto se si guarda all'influenza del modello nordamericano, ma basterà citarne qualcuno per chiarire il discorso: da questo modello alcuni sistemi di civil law hanno derivato la giuria penale (come è accaduto di recente in Spagna), ${ }^{48}$ la tecnica dell'interrogatorio incrociato, l'idea che si debba limitare il ricorso alle corti supreme, la class action, l'impiego di testimonianze scritte sul modello dell'affidavit, e varie altre cose. Non mancano tuttavia, anche se sono assai meno frequenti, ipotesi in cui legislatori di common law hanno guardato ai sistemi dell'Europa continentale: il caso paradigmatico è quello dell'Inghilterra, che ha adottato con le Rules del 1999 l'idea di un codice processuale, fino a quel momento estranea alla tradizione inglese, e che dagli esempi europei sembra aver tratto ispirazione ad es. in materia di misure cautelari e di poteri del giudice.

Un'analisi interessante, ma che qui non può essere neppure accennata, potrebbe riguardare la frequenza, l'intensità e l'importanza relativa degli "scambi" tra sistemi processuali di common law e di civil law. Si può tuttavia accennare in linea generale alla circostanza che i legislatori moderni che si occupano seriamente di riforme della giustizia civile tendono a "prendere a prestito" ciò che ritengono essere utile ai loro scopi senza ritenersi eccessivamente vincolati alle rispettive tradizioni nazionali, e rivolgendosi quindi anche al di fuori dei modelli generali di tradizionale appartenenza.

Naturalmente ciò dipende dalla cultura del legislatore di cui di volta in volta si tratta: un legislatore "colto" avrà le informazioni necessarie per operare scelte migliori sul mercato delle idee relative alla giustizia civile, alle sue finalità e agli strumenti per conseguirle, mentre un legislatore "ignorante " (di cui si danno vari esempi, in particolare in Italia) tenderà ad essere culturalmente autarchico, e quindi a ritenere che il singolo sistema processuale nazionale possa essere riformato solo dall'interno della sua particolare e provinciale cultura, senza alcuna utile influenza derivabile da quegli ordinamenti che hanno già affrontato e risolto - magari meglio ed in modo più efficiente - gli stessi problemi.

Un secondo fattore di evoluzione, strettamente connesso al primo ma da esso distinguibile, può essere indicato in termini molto generali con l'etichetta di conseguenze giuridiche della globalizzazione. Queste conseguenze giuridiche sono numerosissime ed investono molte e diverse aree del diritto, sicchè qui non è neppure possibile tentarne una elencazione, anche perchè lo studio di questi fenomeni è ancora alle battute iniziali, e maggiori approfondimenti occorreranno prima che si possa davvero pensare di aver compreso e defini-

48 Cfr. la Ley organica del Tribunal del Jurado del 22 maggio 1995, su cui v. Comentarios a la Ley del Jurado, coord. Da A. de la Oliva Santos, Madrid 1999. 
to gli effetti che la globalizzazione produce sul piano giuridico. ${ }^{49}$ Limitando il discorso a ciò che può interessare il processo civile, si possono sottolineare due fenomeni particolarmente importanti, che paiono destinati ad assumere rilevanza sempre maggiore. Il primo di questi fenomeni è il rapido e forte incremento della frequenza di controversie transnazionali. Liti coinvolgenti soggetti di diversa nazionalità sono sempre esistite, naturalmente, ma hanno avuto per molto tempo un ruolo tutto sommato marginale rispetto al contenzioso esistente presso le corti dei singoli Stati-nazione. La controversia-tipo, in sostanza, era tipicamente quella infranazionale, ossia quella che coinvolgeva soggetti appartenenti allo stesso ordinamento nazionale. Le cose stanno però cambiando rapidamente, nel senso che diventano sempre più numerose le liti che coinvolgono parti di diversa appartenenza nazionale, in diretta conseguenza del crescere vertiginoso dei rapporti giuridici della natura più diversa (ma soprattutto commerciale e finanziaria) che hanno carattere tipicamente transnazionale. Sotto questo profilo si assiste ad una duplicazione dei "sistemi del mondo" riferibili alla giustizia civile: alla dimensione statale delle controversie, che naturalmente persiste, si sovrappone una diversa dimensione nella quale si colloca il sempre più fitto e frequente intrecciarsi di controversie transnazionali. ${ }^{50}$ Non a caso Habermas parla, per riferirsi a ciò che solitamente si chiama globalizzazione o mondializzazione, di "costellazione postnazionale", ${ }^{51}$ con una metafora che può essere opportunamente usata anche a proposito delle trasformazioni in corso nella giustizia civile.

Il secondo fenomeno che va qui segnalato è la tendenza all'uniformità culturale che comincia a manifestarsi anche nell'ambito della cultura giuridica, oltre che nell'ambito di moltissime aree della cultura generale (letteraria, artistica, cinematografica, musicale, etc.). Anche nella cultura giuridica il superamento dei confini nazionali (o nazionalistici) è un fenomeno ormai inevitabile, oltre che opportuno, ed è strettamente connesso alla diffusione e circolazione di temi di ordine generale come le garanzie costituzionali, i diritti umani, il giusto processo, l'effettività della tutela, e così via. ${ }^{52}$ Se la cultura giuridica, e lo stesso vale per il settore specifico della cultura processualistica, non vuole perdere definitivamente il contatto con la realtà di cui intende occuparsi, e se non vuole rimanere avvolta nella spirale del solipsismo autoreferenziale, deve trovare il modo di partecipare alla circolazione illimitata delle

49 Nella letteratura italiana in argomento vanno comunque segnalati il volume di Maria Rosaria FERRARESE su Le istituzioni della globalizzazione. Diritto e diritti nella società transnazionale, Bologna 2000, e i saggi raccolti in Conflitti e diritti nella società transnazionale, a cura di V.Ferrari, P. Ronfani e S.Stabile, Milano 2001, e in 16 Ragion pratica 2001.

50 Discuto più ampiamente questi fenomeni nel saggio Note sulla dimensione transnazionale delle controversie civili (in corso di stampa). In generale su questa dimensione, sia pure in un'accezione in parte diversa, cfr. anche CAPPELLETTI, Dimensioni della giustizia, cit., p. 39 ss.

51 Cfr. HABERMAS, La costellazione postnazionale, tr.it., Milano 1999.

52 Per una più ampia analisi di questa tendenza, e per riferimenti bibliografici, sia consentito il rinvio a TARUFFO, Dimensioni transculturali, cit., p. 1058 ss. 
idee che ormai è in re ipsa nella dimensione globalizzata del mondo. D'altronde, la deriva postmoderna ci ha ormai abituati a pensare alla cultura non come ad un insieme coerente, chiuso e immobile di contenuti dati una volta per sempre, ma come ad una rete mobile, variabile ed aperta, in continua trasformazione, nella quale molti valori e diversi contenuti, anche tra loro non coerenti o addirittura in conflitto, posso entrare per combinarsi o fondersi in complicati processi di mutamento. Questi fenomeni hanno luogo all'interno dei contesti nazionali in funzione dell'emersione della pluralità di culture anche nell'ambito di singoli sistemi, ma si verificano con particolare intensità su scala globale. ${ }^{53}$ Come la rete transnazionale dei rapporti giuridici allude ad un diritto più "leggero", flessibile, variabile e policentrico, ${ }^{54}$ così la cultura giuridica può e deve diventare meno chiusa e sistematica, più varia e soggetta ad evoluzione, centrata su molti punti di riferimento culturale collocati in vari luoghi del mondo invece che nella tradizione consolidata ed autarchica di un solo paese. Tutto ciò dovrebbe valere, ça va sans dire, anche per ciò che riguarda specificamente il processo civile e quel settore della cultura giuridica che se ne occupa.

\section{Verso nuovi modelli}

Gli ultimi decenni hanno dunque visto verificarsi varie crisi dei modelli ai quali tradizionalmente si riconduceva la distinzione tra sistemi processuali di common law e di civil law. Le trasformazioni che si sono verificate, e che per molti aspetti sono ancora in corso in numerosi ordinamenti, hanno provocato una netta frammentazione dei vecchi schemi e hanno dato luogo a molteplici fenomeni di "ricomposizione" del diritto processuale attraverso un gioco complesso di interferenze tra sistemi diversi, circolazioni di modelli e trapianti di istituti della natura più varia. Si tratta di fenomeni estremamente complicati e di difficile interpretazione, anche perchè essi sono tuttora in progress e non è dato intravederne un momento conclusivo (ammesso che nelle trasformazioni dei sistemi giuridici, e di quelli processuali in particolare, vi sia mai una conclusione). La sola cosa che si può affermare in modo relativamente sicuro è che i consueti e comodi modelli descrittivi, che miravano a rappresentare schematicamente i caratteri fondamentali dei processi di common law e di civil law, appaiono nettamente superati e non sono più utilizzabili come strumenti per conoscere e descrivere i vari ordinamenti.

Ciò non implica tuttavia che non si possa più parlare di modelli processuali come strumenti teorici destinati a servire all'analisi comparatistica oltre che - naturalmente - alla specifica conoscenza dei diversi sistemi. Si tratta però di immaginare modelli diversi da quelli tradizionali, non solo perchè è mutata la realtà che si vorrebbe rappresentare, ma anche perchè, per interpretare questa realtà, sembrano oggi più utili modelli di diversa natura. Sempli-

53 Cfr. per tutti FRIEDMAN, The Horizontal Society, New Haven-London 1999.

54 Cfr. FERRARESE, op. cit., p. $133 \mathrm{ss.}$ 
ficando all'estremo un discorso che dovrebbe essere assai più complesso, si potrebbero immaginare tre tipi fondamentali di modelli processuali: a) modelli strutturali; b) modelli funzionali; c) modelli supranazionali. Vale forse la pena di accennare in estrema sintesi ad alcune delle caratteristiche principali di questa tripartizione.

a) I modelli che possiamo definire come strutturali sono in qualche misura più vicini ai consueti modelli descrittivi, salvo che fanno perno su caratteri "di struttura" del procedimento, invece che a questo o quell'aspetto di discipline processuali nazionali o di ordinamenti appartenenti a specifiche aree geografiche o culturali. Il problema fondamentale è di definire ciò che si intende per struttura di un processo, il che rinvia alla determinazione di quegli aspetti che si considerano fondamentali sulla base di qualche schema culturale, ossia secondo giudizi di valore in funzione dei quali si stabilisce che cosa è importante e si distingue ciò che non lo è. Uno schema culturale che può essere identificato sullo sfondo (o alla base) delle recenti trasformazioni dei principali sistemi processuali porta ad individuare quattro aspetti della struttura del processo che possono considerarsi fondamentali: 1) l'attuazione delle garanzie fondamentali previste nelle varie Costituzioni o Carte dei diritti, e comunque riconosciute a livello nazionale e internazionale (come nell'art.6 della Dichiarazione europea dei diritti dell'uomo o nell'art.47 della Carta europea dei diritti fondamentali), con particolare riferimento alla regola audi et alteram partem e all'indipendenza e imparzialità del giudice; 2) la deformalizzazione e semplificazione della disciplina del processo; 3) l'attribuzione al giudice di funzioni e responsabilità "manageriali" nella direzione del procedimento; 4) l'adozione di uno schema procedimentale a due fasi, l'una destinata alla preparazione (ed eventuale risoluzione anticipata) della causa, l'altra destinata all'assunzione delle prove e alla decisione. I singoli ordinamenti possono evidentemente presentare questi aspetti con intensità diverse e con varie modalità: essi costituiscono comunque punti di partenza per immaginare una "struttura ideale" del processo civile. Un modello articolato su questi quattro aspetti può anche essere utile sia come schema euristico, sia come standard di riferimento per determinare e valutare il grado di evoluzione di ogni ordinamento processuale.

b) I modelli che possiamo definire come funzionali riguardano essenzialmente la strumentalità del processo come mezzo per conseguire i risultati cui è orientata la giustizia civile. Non occorre impegnarsi ora nella definizione di queste finalità, che rinvierebbe alla complessa discussione che riguarda gli scopi istituzionali del processo. Si può ragionevolmente ipotizzare che queste finalità attengano comunque alla risoluzione delle controversie secondo criteri di giustizia, ${ }^{55}$ e muovendo da questa premessa si possono indicare le caratteristiche principali che il processo deve avere per essere funzionale al conseguimento di questo obbiettivo: 1) effettività della tutela

55 Traggo questa sintetica definizione dall'opera più importante in materia di strumentalità e finalità del processo, ossia DINAMARCO, A instrumentalidade do processo, 9. ed., São Paulo 2001, p. 161, alla quale rinvio per un'ampia e approfondita analisi dell'argomento. 
processuale (con particolare riferimento all'accesso alle corti, alla tutela cautelare e alla tutela esecutiva), poichè una protezione inefficace dei diritti equivale a nessuna protezione; 2) rapidità della risoluzione delle controversie (anche e soprattutto prima della conclusione del processo), per l'ovvia ragione che justice delayed is justice denied; 3) adeguatezza specifica del procedimento rispetto alle finalità di tutela delle varie situazioni giuridiche (eventualmente anche attraverso il ricorso a procedimenti differenziati), poichè una tutela inadeguata rispetto alle concrete finalità per le quali essa viene richiesta da chi ne ha bisogno equivale, ancora una volta, a non assicurare alcuna protezione giudiziale dei diritti. Anche sotto il profilo funzionale i singoli ordinamenti concreti possono essere più o meno vicini ad un modello ideale, a seconda del grado e delle modalità con cui forniscono strumenti efficienti per conseguire le finalità essenziali della tutela giurisdizionale. Peraltro tale modello ideale è utile sia per porre a confronto i vari sistemi processuali concreti, sia per stabilire la loro distanza rispettiva dalla situazione che può considerarsi ottimale, sia per formulare valutazioni e configurare progetti di riforma.

c) I modelli supranazionali possono essere strutturali e funzionali, ma si caratterizzano per il fatto di riferirsi a dimensioni che vanno oltre i limiti nazionali dei singoli ordinamenti processuali. Potendo essere strutturali e funzionali, questi modelli possono essere analizzati nei termini ora indicati nei punti a) e b), in maniera simile a quanto può accadere per i modelli nazionali. Tuttavia essi presentano caratteristiche peculiari che portano a distinguere due principali sottocategorie:

1) vi possono essere modelli che riguardano procedimenti appositi per le controversie transnazionali. Un interessante esempio è rappresentato dai Principles and Rules of Transnational Civil Procedure che sono in corso di elaborazione da parte dell'American Law Institute e diUNIDROIT. Questo gruppo di regole è finalizzato a disciplinare in modo uniforme il processo nelle controversie transnazionali (principalmente quelle di natura commerciale), accantonando il tradizionale principio di diritto internazionale della lex fori processuale e rendendo tendenzialmente irrilevante $-\mathrm{al}$ meno in qualche misura - il problema della giurisdizione nazionale e della diversificazione dei sistemi processuali nazionali. ${ }^{56}$

2) Altri modelli si caratterizzano per il fatto di porsi come punti di riferimento per l'unificazione, o almeno l'armonizzazione, delle discipline processuali nazionali dei paesi che appartengono ad ampie aree geografiche e culturali. Questi modelli possono riguardare taluni aspetti particolari del procedimento, oppure possono tendere ad investire l'intero processo civile. Un esempio del primo tipo è rappresentato dal c.d. "progetto Storme", la cui finalità è di unificare la disciplina di alcuni istituti

56 Cfr. Principles and Rules of Transnational Civil Procedure, American Law Institute, Philadelphia 2001 (testo provvisorio). II progetto è stato pubblicato da ultimo, con saggi introduttivi di G.C.Hazard, A.Gidi, R. Stürner e M. Taruffo, in 33 NYU J.Int.L. and Pol. 2001, p. 769 ss.. Per una discussione del progetto cfr. Vers un procés civil universel? Les règles transnationales de procédure civile de l'American Law Institute, Paris, 2001. 
processuali negli ordinamenti dell'area europea $^{57}$. Un importante esempio del secondo tipo è rappresentato dal Codigo Modelo latinoamericano, che si pone appunto come modello per una regolamentazione unificata del processo negli ordinamenti nazionali nell'area dell'America Latina ${ }^{58}$.

Il discorso su questa tipologia di modelli processuali, sulle loro implicazioni e variazioni, e su ciò che si ritiene essenziale per la definizione di ogni singolo modello, richiederebbe evidentemente un'analisi lunga e complessa che qui non può neppure essere avviata. Questi pochi cenni dovrebbero però bastare a far intendere che questi nuovi modelli rappresentano strumenticoncettuali potenzialmente utili per l'analisi, anche comparatistica, degli ordinamenti processuali attuali e futuri. E' all'interno di questi modelli, inoltre, che può essere recuperato il significato residuo della distinzione tra sistemi processuali di common law e di civil law, come modo sintetico per alludere a differenze che hanno avuto un significato storico talvolta considerevole, e che in qualche misura tuttora caratterizzano il processo angloamericano rispetto al processo europeo continentale. Come ho già tentato di chiarire, d'altronde, il nucleo fondamentale delle mie considerazioni non consiste nella negazione delle differenze che pure esistono tra gli ordinamenti processuali che appartengono all'uno piuttosto che all'altro gruppo, ma nella presa d'atto del superamento dei modelli tradizionalmente usati per descrivere e distinguere i due gruppi, e della conseguente necessità di reinterpretare la mutevole realtà dei vari sistemi processuali per mezzo di schemi cognitivamente più aggiornati ed euristicamente più efficaci.

57 Cfr. Rapprochement du droit judiciaire de l'Union européenne - Approximation of judiciary law in the European Union, ed.by M.Storme, Dordrecht 1994.

58 Nella letteratura in lingua italiana in argomento cfr. le relazioni pubblicate nel volume Un "codice tipo" di procedura civile per l'America Latina, a cura di S.Schipani e R. Vaccarella, Padova 1990. E' interessante notare che al Codigo Modelo si fa riferimento anche nei progetti di riforma della giustizia civile nei paesi del Centro Amercica: cfr. FIELD, in FISHER, Legal Reform in Central America. Dispute Resolution and Property Systems, Cambridge, Mass., 2001. 